\title{
Prospecção Científica e Tecnológica do Gênero Pilocarpus Vahl
}

\section{Scientific and Technological Prospection of the Pilocarpus Vahl Genus}

\author{
Cleiton Barroso Bittencourt ${ }^{1}$ \\ Maria Gracelia Paiva Nascimento ${ }^{2}$ \\ Jéssica Cristina Moraes de Araújo ${ }^{3}$ \\ Davi Nascimento Costa ${ }^{3}$ \\ Juelina Oliveira dos Santos ${ }^{3}$ \\ Ivanilza Moreira de Andrade ${ }^{3}$ \\ ${ }^{1}$ Universidade Federal de Lavras, Lavras, MG, Brasil \\ 2Universidade Federal do Piauí, Teresina, PI, Brasil \\ ${ }^{3}$ Universidade Federal do Delta do Parnaíba, Parnaíba, PI, Brasil
}

\begin{abstract}
Resumo
Pilocarpus Vahl é um gênero de espécies arbustivas e arbóreas com centro de diversidade localizado no Brasil, onde apresenta um histórico marcante de utilização médica. Objetivou-se realizar uma prospecção tecnológica e científica em bases de dados nacionais e internacionais sobre Pilocarpus. A metodologia consistiu na busca no título/ resumo dos termos "Pilocarpus" e individualmente as espécies em documentos publicados. Os pedidos de depósitos de patentes tiveram início em 1910 com o maior número de publicações na década de 1990. O Japão e o Brasil lideram a produção tecnológica, incluída, majoritariamente, na subclasse A61K. As espécies Pilocarpus microphyllus e Pilocarpus jaborandi são as mais estudadas e os artigos são direcionados, principalmente, para atividades citotóxicas $e$ antibacteriana. Os dados de distribuição e vulnerabilidade são escassos. Conclui-se que o gênero Pilocarpus é fonte de diversas pesquisas na área médica, e o Brasil apresenta grande potencial biotecnológico com a utilização grupo.
\end{abstract}

Palavras-chave: Biotecnologia. Glaucoma. Jaborandi.

\begin{abstract}
Pilocarpus Vahl is a genus comprising shrub and small tree species with a diversity center located in Brazil, where it presentes a remarkable history of medical use. The objective was to carry out technological and scientific prospection in national and international databases on Pilocarpus. Methodology consisted of searching on title/abstract of the terms "Pilocarpus" and individually the species in published documents. Patent filing applications started in 1910 with the highest number of publications in the 1990s. Japan and Brazil lead the technological production included, mainly, in the subclass A61K. The species Pilocarpus microphyllus and Pilocarpus jaborandi are the most studied and the papers are directed mostly to cytotoxic and antibacterial activities. Distribution and vulnerability data are scarce. It is concluded that the Pilocarpus genus is the source of several researches in the medical field and Brazil has great biotechnological potential with the use of the group.
\end{abstract}

Keyword: Biotecnology. Glaucoma. Jaborandi.

Área Tecnológica: Biotecnologia. 


\section{Introdução}

O Brasil é o país que possui a maior diversidade vegetal do mundo, contabilizando mais de 40 mil espécies de plantas, das quais 10 mil são exclusivas (endêmicas) do território nacional (FIORAVANTI, 2016). Essa ampla fonte de riqueza coloca o país como um dos centros de fornecimento de matéria-prima vegetal, principalmente relacionada ao uso medicinal (OLIVEIRA et al., 2016). Pela viabilidade na obtenção de moléculas bioativas de fontes vegetais, diversas espécies têm sido largamente exploradas pela indústria farmacológica e, por conta disso, pesquisas biotecnológicas têm, cada vez mais, explorado o potencial biotecnológico dessa rica fonte (SILVA et al., 2014; LIMA et al., 2015; ALIYA DEVI; UMA, 2016).

Pilocarpus Vahl (Rutaceae) é um gênero neotropical de plantas arbustivas e arbóreas, com 17 espécies descritas distribuídas na América Central e América do Sul. Para o Brasil estão registradas 15 espécies, das quais 11 são endêmicas (FLORA DO BRASIL 2020, 2020b). Popularmente é conhecido como jaborandi, um dos gêneros mais bem-sucedidos quanto à exploração do potencial medicinal pela indústria farmacêutica (SKORUPA, 2000; PINHEIRO, 2002; SAWAYA et al., 2011). O primeiro registro de seu uso medicinal é reportado em comunidades indígenas da Amazônia, e esse conhecimento tradicional despertou o interesse da comunidade científica no início do século passado para a identificação e isolamento de compostos bioativos (PINHEIRO, 2002; LIMA et al., 2015).

O gênero tornou-se conhecido como fonte natural exclusiva do alcaloide imidazólico pilocarpina, sendo uma das mais importantes drogas para o tratamento do glaucoma e xerostomia, devido à atuação parassimpatomimético colinérgico de ação direta que estimula a secreção das glândulas exócrinas, glândulas mucosas salivares, sudoríparas, lacrimais e respiratórias (MALAYSIA, 2014; LIMA et al., 2015). Na década de 1970, os medicamentos utilizados para o tratamento do glaucoma à base de pilocarpina representavam cerca de $70 \%$ no mercado mundial (FILHO et al., 2003) e, mesmo com a entrada de novos fármacos com propriedades semelhantes, a pilocarpina ainda vem participando na formulação de mióticos (BITTENCOURT et al., 2018a).

Além do metabólito secundário pilocarpina, das folhas dessas espécies já foram extraídas, purificadas e identificadas também a presença de isopilocarpina, isopilocarpidina, pilosina, entre outros, contudo, apenas a pilocarpina é comercializada e utilizada na terapêutica (ABREU et al., 2011; SAWAYA et al., 2011; VERAS et. al., 2012). Nos últimos anos houve crescimento do número de pesquisas sobre pilocarpina (BITTENCOURT, 2018a) e outros metabólitos presentes no gênero vêm tendo grande destaque em pesquisas como, por exemplo, de atividade esquistossomicida (VERAS et al, 2012; GUIMARÃES et al., 2018).

É importante destacar que por volta de $25 \%$ dos medicamentos comercializados atualmente são de origem vegetal, e este fato enfatiza a ampla importância de se conhecer as atividades farmacológicas de espécies com histórico de uso médico como Pilocarpus (KALLUF, 2008; ALIYA; DEVI; UMA, 2016). Dessa forma, apresentar dados prospectivos com base em artigos e tecnologias produzidas podem fornecer subsídios interessantes, além de direcionar várias vertentes do desenvolvimento técnico científico, uma vez que estas informações demonstram a evolução e inovação dos conhecimentos sobre determinada área, influenciando positivamente a indústria, economia ou a sociedade de um modo geral (KUPFER; TIGRE, 2004). 
Dessa forma, objetivou-se com este trabalho realizar uma prospecção tecnológica e científica acerca das espécies do gênero Pilocarpus, apresentando um panorama de aplicações terapêuticas, número de publicações e de depósitos de patentes em bancos nacionais e internacionais e dados de vulnerabilidade das espécies.

\section{Materiais e Métodos}

A pesquisa foi realizada em fevereiro de 2020 utilizando-se como palavras-chave "Pilocarpus" e as espécies com os nomes validados de ocorrência no Brasil e no mundo (exemplo: "Pilocarpus microphyllus") nos bancos de artigos e patentes publicadas de 1910 a 2020, sendo considerados válidos os documentos que apresentassem esses termos no título e/ou resumo. Para realizar a prospecção tecnológica foram consultados os seguintes bancos de patentes: World Intellectual Property Organization (WIPO), European Patent Office (EPO) e o Instituto Nacional da Propriedade Industrial do Brasil (INPI). As patentes foram analisadas quanto ao país depositante, o ano do pedido depósito e sua Classificação Internacional de Patentes (CIP). A prospecção científica foi obtida nas bases de artigos Scopus e Web of Science, e os documentos foram analisados quanto ao ano de publicação, área de aplicação e distribuição por espécie. A opção pela utilização das respectivas bases de artigos e patentes se deu pela confiabilidade e versatilidade dos dados disponíveis e, principalmente para os artigos científicos, por reunirem um grande número de publicações.

Para o levantamento sobre espécies de Pilocarpus ocorrentes no Brasil e no mundo foram realizadas buscas no sistema Flora do Brasil 2020 (2020b); para as espécies que apresentaram sinônimos foram feitas consultas no texto dos artigos a fim de esclarecer a espécie em questão pesquisada com finalidade terapêutica. Para avaliar o índice de vulnerabilidade e o endemismo das espécies foram consultados o Livro Vermelho da Flora do Brasil (MARTINELLI; MORAES, 2013), o Centro Nacional de Conservação da Flora (CNCFLORA, 2020) e o sistema Flora do Brasil 2020 em construção (FLORA DO BRASIL 2020, 2020a). Os dados foram tabulados de forma individual e organizados em tabelas e gráficos no Microsoft Excel (2019).

\section{Resultados e Discussão}

Ao analisar os dados tecnológicos com a palavra-chave Pilocarpus (Tabela 1), um total de 71, 28 e 9 documentos foram depositados nos bancos de dados World Intellectual Property Organization (WIPO), European Patent Office (EPO) e Instituto Nacional da Propriedade Intelectual (INPI), respectivamente. O maior número de artigos indexados deve-se ao banco de resumos e citações Scopus, com 130 registros, enquanto na Web of Science obteve-se um total de 76 artigos indexados. 
Tabela 1 - Indicadores botânicos (nível de endemismo e índice de vulnerabilidade) e prospecção científica e tecnológica de espécies pertencentes ao gênero Pilocarpus que apresentaram patentes registradas nos bancos de dados consultados

\begin{tabular}{|c|c|c|c|c|c|c|c|}
\hline \multirow{2}{*}{ ESPÉCIES/DESCRITORES } & \multicolumn{2}{|c|}{$\begin{array}{l}\text { INDICADORES } \\
\text { BotÂNICOS }\end{array}$} & \multicolumn{3}{|c|}{$\begin{array}{l}\text { Prospecção } \\
\text { TeCnOlóGica }\end{array}$} & \multicolumn{2}{|c|}{ Prospecção Científica } \\
\hline & END & IDV & WIPO & EPO & INPI & Scopus & $\begin{array}{l}\text { Web of } \\
\text { ScIENCE }\end{array}$ \\
\hline Pilocarpus & $\mathrm{N}$ & - & 71 & 28 & 9 & 130 & 76 \\
\hline Pilocarpus alatus & $S$ & $\mathrm{VU}$ & - & - & - & 3 & 3 \\
\hline Pilocarpus carajaensis & $S$ & $\mathrm{NE}$ & - & - & - & 2 & 1 \\
\hline Pilocarpus demerare & $\mathrm{N}$ & $\mathrm{NE}$ & - & - & - & - & - \\
\hline Pilocarpus giganteus & $S$ & NT & - & - & - & - & - \\
\hline Pilocarpus grandifolius & S & $\mathrm{NE}$ & - & - & - & - & - \\
\hline Pilocarpus jaborandi & S & EN & 18 & 17 & 5 & 50 & 33 \\
\hline Pilocarpus manuensis & $\mathrm{N}$ & $\mathrm{NE}$ & - & - & - & 1 & 1 \\
\hline Pilocarpus microphyllus & $\mathrm{N}$ & EN & 6 & 5 & 1 & 38 & 31 \\
\hline Pilocarpus pauciflorus & $S$ & $\mathrm{NE}$ & - & - & - & 2 & 1 \\
\hline Pilocarpus pennatifolius & $\mathrm{N}$ & $\mathrm{NE}$ & 1 & 2 & - & 14 & 10 \\
\hline Pilocarpus longeracemosus & S & $\mathrm{NE}$ & - & - & - & 0 & - \\
\hline Pilocarpus peruvianus & $\mathrm{N}$ & $\mathrm{NE}$ & - & - & - & 1 & 1 \\
\hline Pilocarpus riedelianus & S & $\mathrm{NE}$ & - & - & - & 3 & 1 \\
\hline Pilocarpus spicatus & S & $\mathrm{NE}$ & - & - & - & 17 & 13 \\
\hline Pilocarpus sulcatus & $S$ & $\mathrm{NE}$ & - & - & - & 1 & 1 \\
\hline Pilocarpus trachylophus & S & EN & - & - & - & 2 & 2 \\
\hline Pilocarpus trifoliolatus & S & $\mathrm{NE}$ & - & - & - & 1 & 1 \\
\hline Total & - & - & 96 & 52 & 14 & 265 & 175 \\
\hline
\end{tabular}

Legendas: $\mathrm{END}=$ Nível de endemismo; $\mathrm{S}=$ Endêmica do Brasil; $\mathrm{N}=$ Não endêmica; IDV= Índice de vulnerabilidade; NE= Não avaliada quanto à ameaça de extinção; $\mathrm{EN}=$ Em perigo; $\mathrm{NT}=$ Quase ameaçada; $\mathrm{VU}=$ Vulnerável

Fonte: Elaborada pelos autores deste artigo (2020)

Entre as espécies utilizadas como descritores nos bancos de patentes (Tabela 1), apenas Pilocarpus jaborandi Holmes, Pilocarpus microphyllus Stapf ex Wardlew ePilocarpus pennatifolius Lem tiveram tecnológicas registradas, representando um total de 17,64\% das espécies do grupo. Quanto aos dados de artigos científicos, $82,35 \%$ das espécies foram fonte de pesquisas com divulgação para a comunidade científica. O maior número de trabalhos publicados deve-se à espécie Pilocarpus jaborandi, com $37,03 \%$ dos registros $(n=50)$, seguidos de Pilocarpus microphyllus ( $\mathrm{n}=38$ ), Pilocarpus spicatus A.St.-Hil $(\mathrm{n}=17)$ e Pilocarpus pennatifolius $(\mathrm{n}=)$, indexados na Scopus.

Pilocarpus é um dos gêneros comercialmente importantes da família Rutaceae pelo fato de ser fonte natural e exclusiva de pilocarpina, alcaloide utilizado no tratamento do glaucoma (MALAYSIA, 2014; LIMA et al., 2015), além de ter suas folhas reconhecidas como potente estimulante da salivação e da transpiração (CALDEIRA et al., 2017). P. microphyllus é a espécie que 
possui o maior teor de pilocarpina em suas folhas, fator que impulsiona interesse em pesquisas que buscam novas biomoléculas com atividades terapêuticas para esta espécie (VERAS et al., 2012; SILVA et al., 2014; ROCHA et al., 2017). Quanto a P. jaborandi, que lidera as pesquisas que resultaram em publicações, registra-se um histórico de uso pela medicina tradicional em comunidades indígenas da Amazônia, o que popularizou o jaborandi, fato este que influencia no grande número de estudos e citações em resumos e títulos (CALDEIRA et al., 2017). Mesmo com poucas tecnologias e artigos registrados para outras espécies do grupo, a pilocarpina foi registrada para quase todos os representantes. Além disso, outros metabólitos secundários com atividade biológica foram recentemente reportados para o grupo, o que vem a agregar valor pelo potencial medicinal futuro (LIMA et al., 2015; CASTRO et al., 2016).

Segundo dados da Flora do Brasil 2020 (2020b), em território nacional foi catalogado o maior número de espécies $(n=15)$ com seu centro de diversidade situado nas regiões Norte e Nordeste do Brasil, em especial no estado do Maranhão, o qual, desde a segunda metade do século passado, é o grande centro de coletas de folhas para a produção de sais de pilocarpina (PINHEIRO, 2002). É notória a preocupação em relação à conservação do grupo, visto que o país apresenta altos índices de desmatamento e planejamento ineficiente em relação às suas reservas ecológicas (BANDEIRA; DEIMILING; GEORG-KRAEMER, 2010), o que tem levado à redução do habitat de espécies importantes como resultado de incêndios, corte de árvores para fins comerciais, devastação de terras para utilização da agropecuária ou, até mesmo, fenômenos naturais (ARRAES; MARIANO; SIMONASSI, 2012, fator este que expõe as espécies de Pilocarpus à redução substancial das suas populações (BANDEIRA; DEIMLING; GEORG-KRAEMER, 2010).

Entre as 15 espécies do grupo ocorrentes no Brasil, apenas quatro (Pilocarpus alatus, Pilocarpus giganteus, Pilocarpus jaborandi e Pilocarpus microphylluys) apresentam dados quanto aos índices de vulnerabilidade e de risco de extinção (NE), incluídas nos critérios pela União Internacional para a Conservação da Natureza (IUCN, 2012; FLORA DO BRASIL 2020, 2020b). Visto o Brasil ser o centro de endemismo do gênero $(n=11)$ (CALDEIRA et al., 2017), é preocupante a falta de informações a respeito da vulnerabilidade das espécies de Pilocarpus, o que, de acordo com Cruz et al. (2016), evidenciaria os possíveis efeitos negativos sobre o grupo advindos de pressões sobre a capacidade adaptativa. Mesmo com a falta de dados ecológicos, é reportado que Pilocarpus spp. sofrem acentuado extrativismo, principalmente das espécies ocorrentes nas regiões Norte e Nordeste do Brasil (PINHEIRO, 2002), o que levou a inclusão de Pilocarpus microphyllus na Lista Vermelha da Flora do Brasil em 2013. Tendo em vista a grande diversidade vegetal, a falta de informação sobre a distribuição e o atual cenário de conservação colocam em risco espécies como as de Pilocarpus spp., com grande potencial na área médica (RODRIGUES; BARBOSA, 2012).

O número de depósitos de patentes e artigos indexados nos bancos de dados do WIPO, EPO e Scopus obtidos com o descritor Pilocarpus no período de 1910-2020 está ilustrado na Figura 1, onde é possível observar que as publicações de artigos tiveram início em 1910 ( $n=1)$ e intensificaram-se a partir do ano de 1990, com maior número de publicações em 2018 ( $n=8)$. Tendo em vista as patentes depositadas na WIPO, os registros iniciaram-se em 1989 ( $n=4)$, com maior número de pedidos nos anos de $1997(n=8)$ e $2004(n=9)$. Na EPO foram registrados 28 documentos, com início em 1920 e com o maior número de publicações registrado em meados de 1997. 
Figura 1 - Número de depósitos de patentes obtido, utilizando-se como descritor Pilocarpus, nos bancos de dados World Intellectual Property Organization (WIPO), European Patent Office (EPO) e número de publicações científicas em consulta ao acervo Scopus entre 1910 a 2020

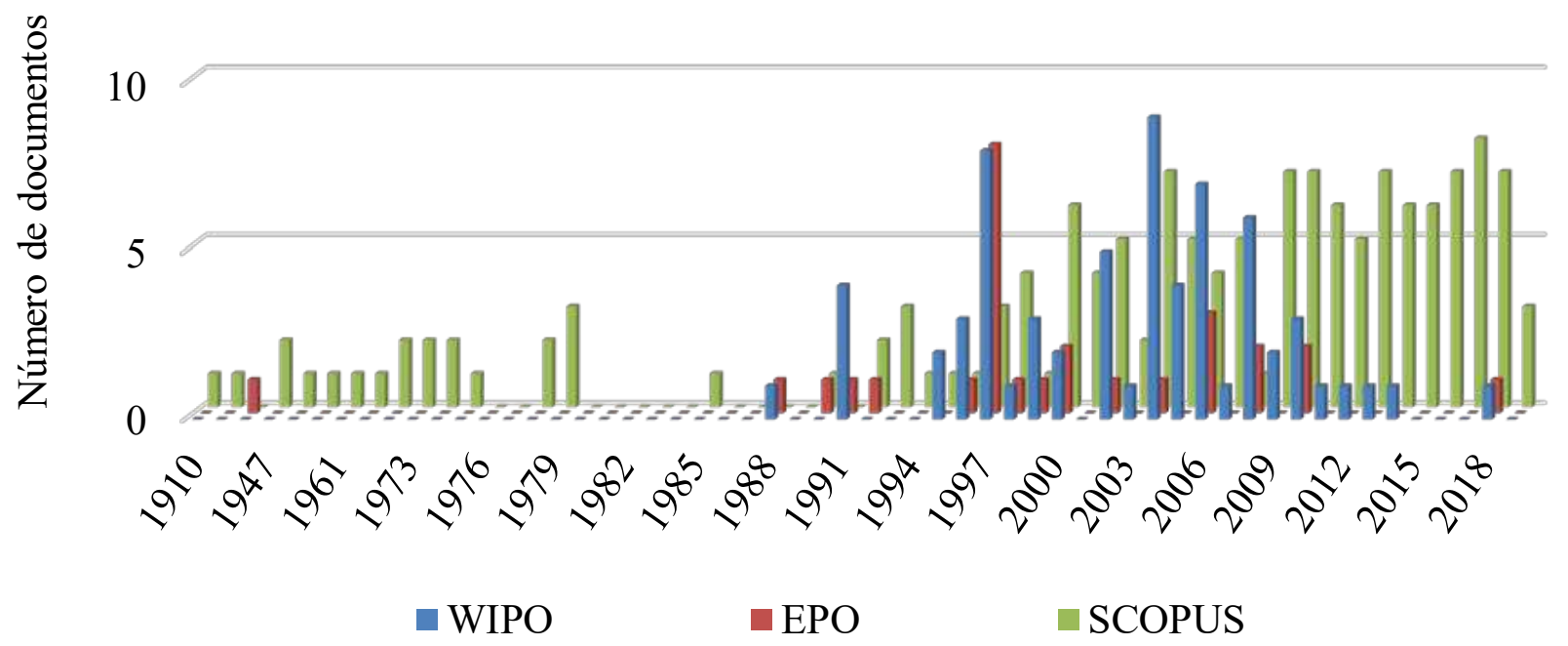

Fonte: Elaborada pelos autores deste artigo (2020)

Devido à aplicação de Pilocarpus no tratamento do glaucoma, a partir de 1988, quando se intensificaram os depósitos de patentes, as pesquisas que geraram tecnologias tiveram como principal foco de estudo a pilocarpina para a produção de extratos, estabilização de alcaloides e preparação de pilocarpina em cultura in vitro (WIPO, 2020). No início dos anos 2000, os depósitos seguiram a linha de pesquisas com o uso de moléculas vegetais com atividade anticâncer (BITTENCOURT et al., 2018b) e os depósitos sobre Pilocarpus concentraram-se nessa área até 2004, na qual a proteína heteropilocarpina, extraída de Pilocarpus spp., foi adaptada para a preparação de medicamento destinado ao tratamento de câncer, em particular, no tratamento do cancro de pulmão e de mama. É sabido que o câncer é uma das mais complexas doenças que afetam milhões de pessoas no mundo e drogas advindas de plantas são, em suma, agentes promissores para o seu tratamento por, além de apresentarem alta afinidade com um alvo biológico, possuírem potente atividade para inibir a metástase (MANS; ROCHA; SCHWARTSMANN, 2000; ALIYA; DEVI; UMA, 2016).

O Japão e o Brasil destacam-se como os maiores detentores de pedidos de patentes registradas envolvendo o desenvolvimento de tecnologias com o gênero Pilocarpus, com 27 e 12 patentes, respectivamente (Figura 2). O Japão é um dos países que mais promovem inovações tecnológicas no mundo e está entre os 20 primeiros colocados em ranking divulgado pela Organização Mundial da Propriedade Intelectual (OMPI) (OMS, 2018). Por possuir a maior biodiversidade vegetal do planeta e ser o centro de diversidade do gênero, o Brasil apresenta grande potencial inovador na área de produtos naturais com a finalidade biotecnológica. Além disso, os dados apontam que o país concorre com nações que apresentam um histórico de inovação, como Japão e Estados Unidos, em pesquisas sobre o gênero. 
Figura 2 - Distribuição dos depósitos por países com o gênero Pilocarpus nos bancos de dados World Intellectual Property Organization (WIPO), European Patent Office (EPO) e Instituto Nacional da Propriedade Industrial (INPI) de 1910 a 2020

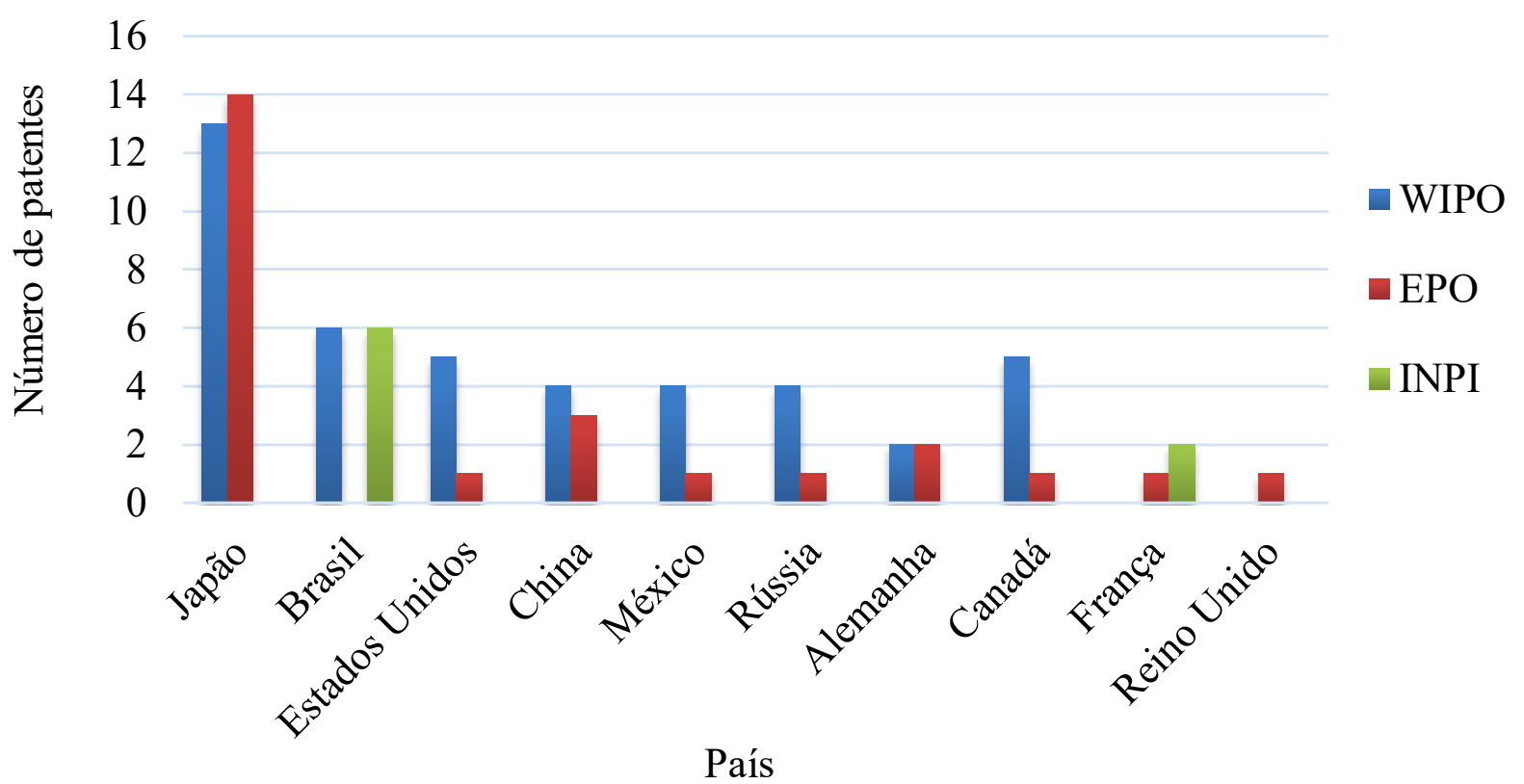

Fonte: Elaborada pelos autores deste artigo (2020)

Analisando-se os documentos conforme a CIP (Figura 3), é possível observar que a seção A (Necessidade Humana) possui o maior número de depósitos, seguida pela seção C (química e metalurgia). É importante destacar que na pesquisa foram encontrados 108 documentos, porém, estes são classificados em 50 CIPS e podem ter uma ou mais subclasses para caracterizá-lo (WIPO, 2020). Entre os pedidos depósitos de patentes registrados nas bases internacionais, 86 estão alocados na subclasse $\mathrm{A} 61 \mathrm{~K}$, que trata de preparações para finalidades médicas, odontológicas ou higiênicas. Além disso, um número expressivo de patentes (45, no total) está inserido na subclasse A61P, que está relacionado à atividade terapêutica específica de compostos químicos ou preparações medicinais, e na subclasse A61Q (30 patentes), que aborda compostos com uso específico de cosméticos ou preparações similares para higiene pessoal. Outras patentes, em menor número, estão alocadas nas subclasses C07K (peptídeos), C12N (engenharia genética ou de mutações e meios de cultura) e $\mathrm{C} 12 \mathrm{P}$ (processos de fermentação ou processos que utilizem enzimas para sintetizar uma composição ou composto químico desejado). Na base nacional INPI foram registrados pedidos majoritariamente voltados para a área médica (A61K e A61Q), seguidos de uso na obtenção de composição de cosméticos (A61K). 
Figura 3 - Distribuição por CIP dos depósitos com o gênero Pilocarpus nos bancos de dados World Intellectual Property Organization (WIPO), European Patent Office (EPO) e no Instituto Nacional da Propriedade Industrial (INPI) de 1910 a 2020

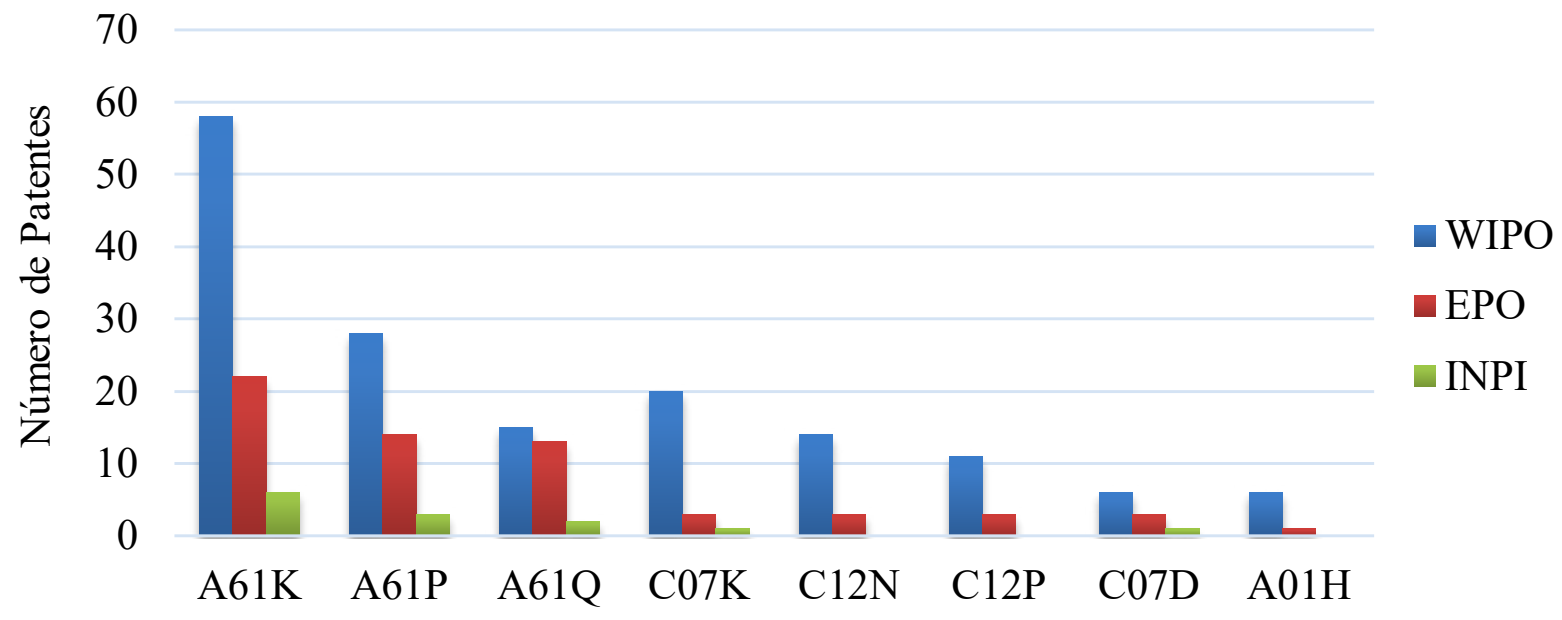

Classificação Internacional de Patentes (CIP)

Fonte: Elaborada pelos autores deste artigo (2020)

Nas publicações indexadas de 1910 a 2020 na base de artigos Scopus (Figura 4) observa-se que 13 das 17 espécies descritas para o gênero (87,50\%) apresentam pesquisas que resultaram em publicações de artigos indexadas, no entanto, o maior número de publicações tem como fonte de estudo as espécies $P$. jaborandi (50), P. microphyllus (38), P. spicatus (17) e P. pennatifolius (14), representando $88,14 \%$ do total de publicações. $P$. pennatifolius foi a primeira espécie com artigo indexado na base Scopus em 1910, em um estudo da anatomia foliar (TUNMANN; JENZER, 1910) e, com o reconhecimento da atividade farmacológica do grupo, seguiram-se estudos que envolveram a presença de pilocarpina (SALLES et al., 2004), análises do conteúdo voláteis (SANTOS, 2004) e, recentemente, fitoquímica e atividade antimicrobiana (CARMO et al., 2018). Comparado a outras espécies, $P$. spicatus teve a primeira pesquisa indexada na Scopus somente em 1992, em um estudo que envolveu a aplicação de óleo essencial para atividade edemática (SILVA; RAO, 1992). Os estudos sobre P. jaborandi e P. microphyllus, pelo grande número de documentos indexados, foram mais diversificados e os primeiros envolveram o isolamento e identificação de diversos metabólitos secundários como, por exemplo, da pilosina, isopilosina e epiisopilosina (LÖWE; POOK, 1973; TEDESCHI et al., 1973). Além da evolução do conhecimento sobre constituintes químicos das espécies ao longo do século passado, que foi impulsionado pela utilização intensiva da pilocarpina no tratamento do glaucoma, autores, a partir dos anos 2000, discutiram também os possíveis impactos do extrativismo de folhas, $e$ estudos de diversidades foram realizados com o objetivo de apontar o impacto da atividade sobre populações naturais e direcionar estratégias de proteção (MOURA, 2005; SIDHU, 2014). 
Figura 4 - Número de publicações das principais espécies do gênero Pilocarpus indexadas no banco de dados do Scopus de 1910 a 2020

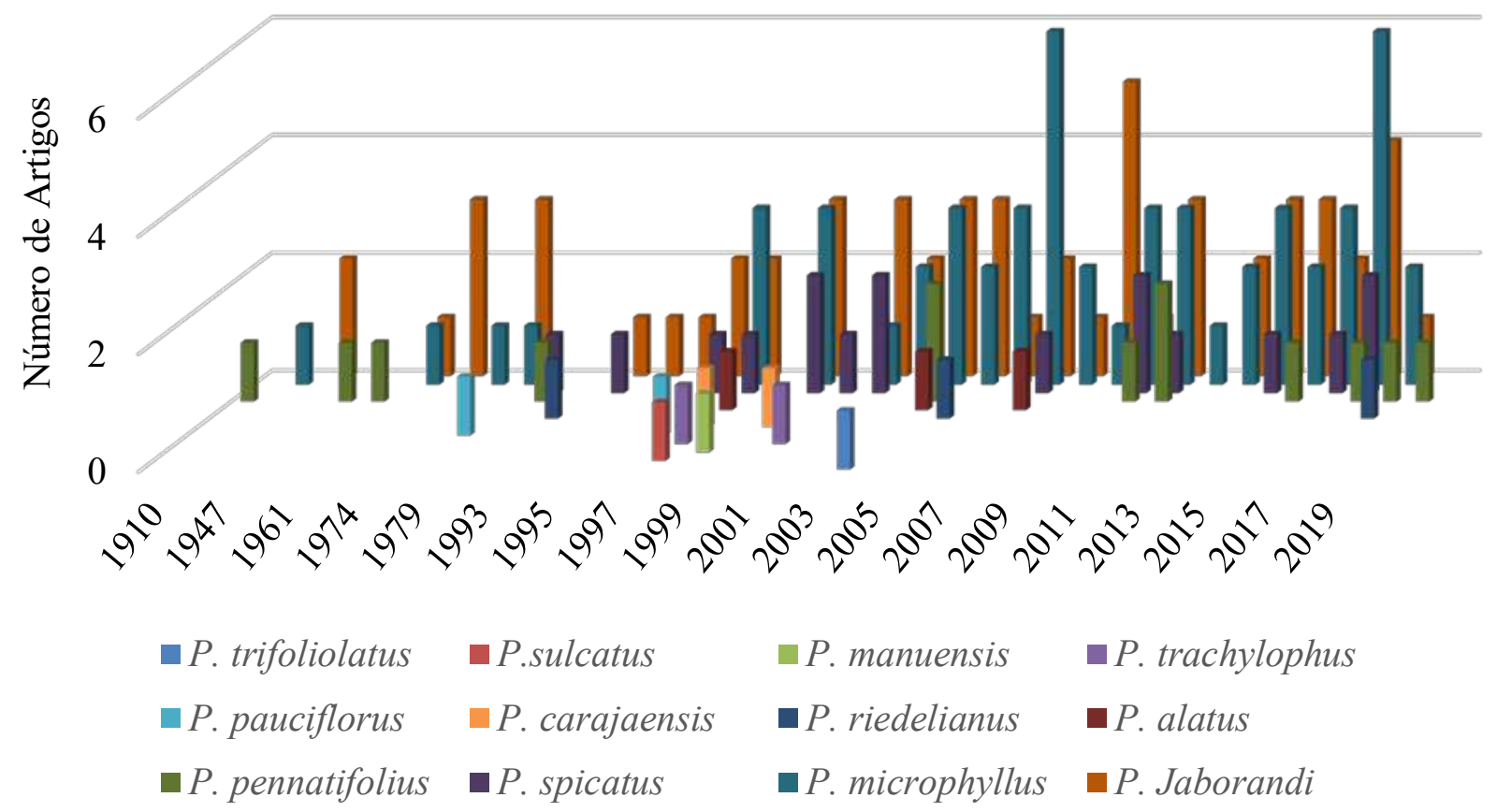

Fonte: Elaborada pelos autores deste artigo (2020)

As pesquisas sobre o gênero no início do século passado voltaram-se para a descrição de metabólitos secundários, principalmente da classe dos alcaloides (VYSOTSKAIA; MUSHINSKAIA; SHOSTENKO, 1966; LINK; BERNAUER; OBERHÄNSLI, 1974). Os estudos que se seguiram buscaram a presença de outros constituintes secundários, como cumarinas e óleos essenciais que foram reportados para o grupo (AMARO-LUIS et al., 1990). Sabe-se que os metabólitos secundários estão diretamente envolvidos nos mecanismos que permitem adaptação dos vegetais ao meio em que vivem (PEREIRA; CARDOSO, 2012; CAMPOS et al., 2016) e, geralmente de estrutura complexa, esses produtos vegetais apresentam marcante atividade biológica (SIMÕES et al. 2007). Um dos alcaloides mais utilizados atualmente é a morfina, que atua como analgésico em pacientes com câncer em fase terminal (NOGUEIRA; SAKATA, 2012), e as cumarinas, que atuam também como analgésico além de anti-helmíntico, anti-HIV e diurético, entre outras atividades (WATERMAN, 1975).

As buscas por princípios ativos com espécies de Pilocarpus abrangeram em maior frequência a verificação da propriedade citotóxica (28\%), seguida de teste de atividade antibacteriana (21\%) e atividade antimicrobiana (15\%) (Figura 5). O uso das propriedades nocivas a células das substâncias presentes no gênero teve como via de aplicação a atividade contra Schistosomose (VERAS et al., 2012; GUIMARÃES et al., 2018), a atividade antihelmintica (ROCHA et al., 2017) e também a ação leishmanicida (MARIN et al., 2018). Outro uso marcante foi sua atividade contra microrganismos patógenos, principalmente as bactérias, pela sua resistência às drogas comercializadas e por afetar a saúde de milhões de pessoas ao redor do mundo (GUERREIRO et al., 2005; ARARUNA et al., 2012). 
Figura 5 - Número de publicações sobre o gênero Pilocarpus indexadas no banco de dados do Scopus, de 1910 a 2020, de acordo com a finalidade terapêutica

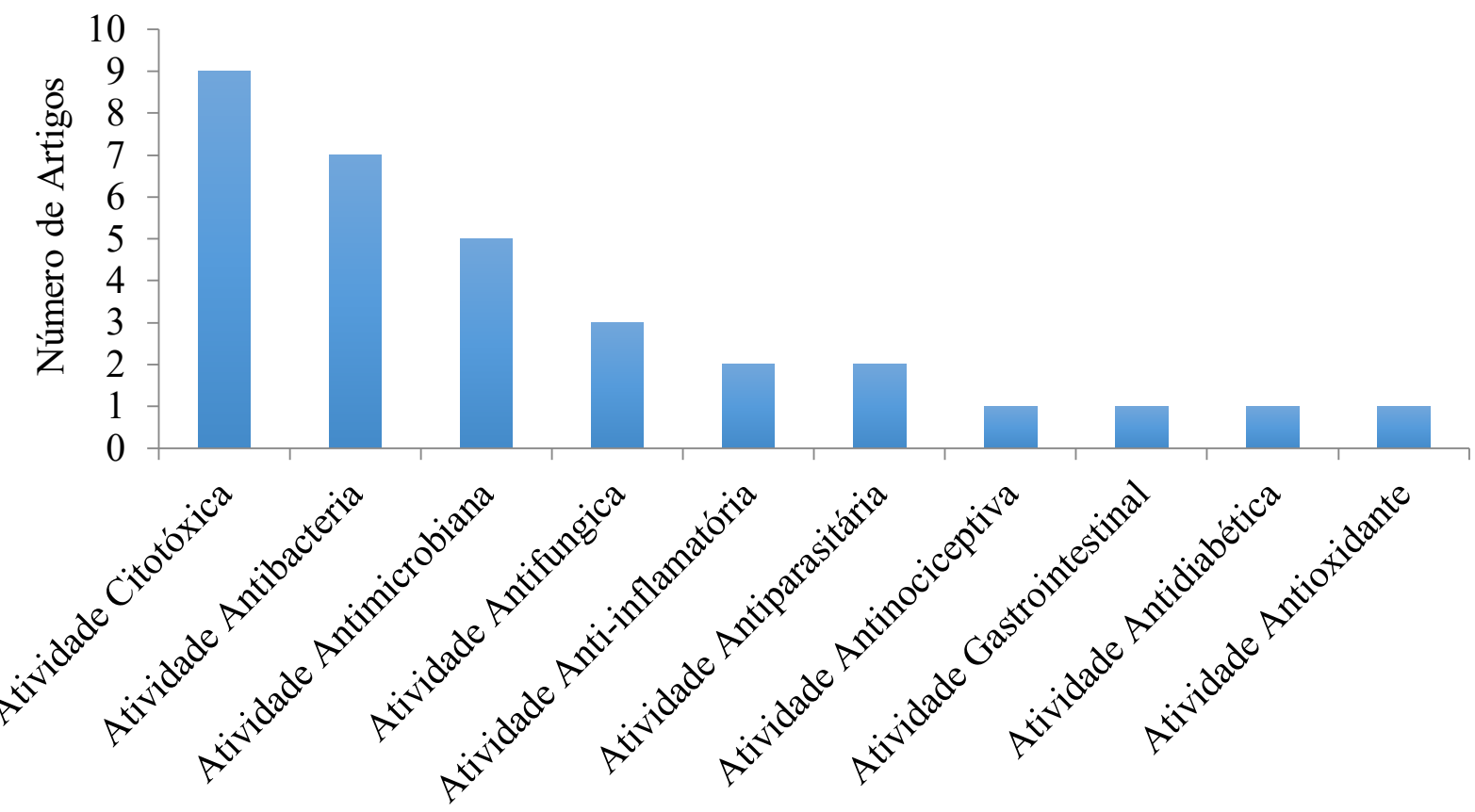

Atividade Pesquisada

Fonte: Elaborada pelos autores deste artigo (2020)

\section{Considerações Finais}

As pesquisas sugerem que o gênero Pilocarpus tornou-se conhecido devido às suas propriedades como estimulante do sistema nervoso parassimpático, tendo como princípio ativo o alcaloide pilocarpina, largamente utilizado para tratamento de disfunções oculares como o glaucoma. O Japão e o Brasil foram os maiores detentores de pedidos de patentes, majoritariamente registrados na década de 1990. Além disso, houve o crescimento de publicações científicas sobre o grupo, com P. jaborandi e P. microphyllus despontando como as espécies com o maior número de tecnologias e artigos produzidos. As pesquisas tiveram como principal objetivo o uso das espécies para finalidade médica, incluindo principalmente atividade citotóxica e antibacteriana. Mesmo com o histórico de utilização do grupo, algumas espécies encontram-se vulneráveis e a maioria não possui indicadores de conservação publicados. Com isso, destaca-se o grande potencial do Brasil em pesquisas médicas com Pilocarpus spp. e a necessidade de promover esforços para a conservação dessas espécies.

\section{Referências}

ABREU, Ilka N. et al. Metabolic alterations in different developmental stages of Pilocarpus microphyllus. Planta Médica, [S.I.], v. 77, n. 3, p. 293-300, 2011.

ALIYA, Sheik; DEVI, Yalavarthy Prameela; UMA, Adepelly. Plants as potential resources of anticancer drugs. Current Trends in Biotechnology and Pharmacy, [S.I.], v. 10, p. 92-107, 2016. 
AMARO-LUIS, Juan M. et al. New coumarins from Pilocarpus goudotianus. Planta Médica, [S.l.], v. 56, n. 3, p. 304-306, 1990.

AMPARO, Keize Katiane dos Santos; RIBEIRO, Maria do Carmo Oliveira; GUARIEIRO, Lilian Lefol Nani. Estudo de caso utilizando mapeamento de prospecção tecnológica como principal ferramenta de busca científica. Perspectivas em Ciência da Informação, [S.I.], v. 17, n. 4, p. 195-209, 2012.

ARARUNA, Mariana KA et al. Evaluation of antibiotic \& antibiotic modifying activity of pilocarpine \& rutin. The Indian Journal of Medical Research, [S.I.], v. 135, n. 2, p. 252, 2012.

ARRAES, Ronaldo de Albuquerque; MARIANO, Francisca Zilania; SIMONASSI, Andrei Gomes. Causas do desmatamento no Brasil e seu ordenamento no contexto mundial. Revista de Economia e Sociologia Rural, [S.I.], v. 50, n. 1, p. 119-140, 2012.

BANDEIRA, Ana Júlia; DEIMLING, Luis Irineu; GEORG-KRAEMER, Janaína Endres. Variabilidade genética do jaborandi (Pilocarpus pennatifolius Lemaire; Rutaceae) em populações naturais da região noroeste do Rio Grande do Sul. Revista de Iniciação Científica da ULBRA, [S.1.], n. 8, 2010.

BITTENCOURT, Cleiton Barroso et al. Prospecção tecnológica e científica do uso da pilocarpina no tratamento do glaucoma. Revista Interdisciplinar de Ciências Médicas, Teresina, anual, 2018a.

BITTENCOURT, Cleiton Barroso et al. Technological prospection of Alkaloids used as alternative route in cancer treatment. Revista GEINTEC-Gestão, Inovação e Tecnologias, [S.I.], v. 8, n. 4, p. 4.620-4.631, 2018b.

CALDEIRA, Cecilio Frois et al. Sustainability of Jaborandi in the eastern Brazilian Amazon. Perspectives in Ecology and Conservation, [S.I.], v. 15, n. 3, p. 161-171, 2017.

CAMPOS, S. C. et al. Toxicidade de espécies vegetais. Revista Brasileira de Plantas Medicinais, [S.l.], v. 18, n. 1, p. 373-382, 2016.

CARMO, Gabriele et al. Phytochemical and antimicrobial study of Pilocarpus pennatifolius Lemaire. Fitoterapia, [S.l.], v. 131, p. 1-8, 2018.

CASTRO, Karina Neoob de Carvalho et al. In vitro effects of Pilocarpus microphyllus extracts and pilocarpine hydrochloride on Rhipicephalus (Boophilus) microplus. Revista Brasileira de Parasitologia Veterinária, [S.I.], v. 25, n. 2, p. 248-253, 2016.

CNCFLORA. Pilocarpus. [2020]. Disponível em: http://cncflora.jbrj.gov.br/portal. Acesso em: 10 fev. 2020.

CRUZ, Maria João et al. Assessing biodiversity vulnerability to climate change: testing different methodologies for Portuguese herpetofauna. Regional Environmental Change, [S.l.], v. 16, n. 5, p. 1.293-1.304, 2016.

EPO - EROPEAN PATENT OFFICE. [2018]. Disponível em: https://worldwide.espacenet.com/ advancedSearch?locale $=$ en_EP. Acesso em: 10 feb. 2018.

FIORAVANTI, Carlos. A maior diversidade de plantas do mundo. Pesquisa FAPESP, São Paulo, n. 241, p. 42-47, 2016.

FLORA DO BRASIL 2020. Pilocarpus Vahl. [2020a]. Disponível em: http://floradobrasil.jbrj.gov.br/ reflora/floradobrasil/FB866. Acesso em: 10 fev. 2020.

FLORA DO BRASIL 2020. Programa REFLORA. Jardim Botânico do Rio de Janeiro. [2020b].

Disponível em: http://floradobrasil.jbrj.gov.br. Acesso em: 15 fev. 2020 
GUERREIRO, Gisleine et al. Sesquiterpenos do caule de Pilocarpus riedelianus e atividades sobre micro-organismos. Química Nova, [S.1.], v. 28, n. 6, p. 986-990, 2005.

GUIMARÃES, Maria A. et al. Epiisopilosine alkaloid has activity against Schistosoma mansoni in mice without acute toxicity. PloS One, [S.I.], v. 13, n. 5, p. e0196667, 2018.

INPI - INSTITUTO NACIONAL DA PROPRIEDADE INDUSTRIAL. Informação tecnológica: Busca de patentes. [2020]. Disponível em http://www.inpi.gov.br/menu-servicos/informacao/busca-depatentes. Acesso em: 10 fev. 2020

IUCN - INTERNATIONAL UNION FOR CONSERVATION OF NATURE. IUCN red list categories and criteria. 2012. Disponível em: https://portals.iucn.org/library/sites/library/files/documents/RL2001-001-2nd.pdf. Acesso em: 10 fev. 2020.

KALLUF, Lucyanna de J. H. Fitoterapia funcional: dos princípios ativos à prescrição de fitoterápicos. In: KALLUF, Lucyanna de J. H. Fitoterapia funcional: dos princípios ativos à prescrição de fitoterápicos. [S.I.]: [s.n.], 2008. p. 304-304.

KUPFER, David; TIGRE, Paulo Basto. Prospecção Tecnológica. In: CARUSO, L. A.; TIGRE, P. B. (org.). Modelo SENAI de prospecção: documento metodológico. Montevideo: OIT/CINTERFOR, 2004. p. 17-29.

KIM, Jung-Hoon et al. Camptothecin and topotecan inhibit adipocyte differentiation by inducing degradation of PPAR $\square$. Biochemical and biophysical research communications, [S.I.], v. 463, n. 4, p. 1.122-1.128, 2015.

LIMA, David Fernandes et al. Prospecção Tecnológica do Jaborandi (Pilocarpus microphyllus): espécie economicamente importante no Norte e Nordeste do Brasil. Revista GEINTEC - Gestão, Inovação e Tecnologias, [S.l.], v. 5, n. 1, p. 1.626-1.638, 2015.

LINK, Helmut; BERNAUER, Karl; OBERHÄNSLI, Willi E. Configuration of Pilocarpus alkaloids. Helvetica Chimica Acta, [S.l.], v. 57, n. 7, p. 2.199-2.200, 1974.

LISTA VERMELHA. Índices de vulnerabilidade. [2010]. Disponível em: . Acesso em: 10 jan. 2020.

LÖWE, Wilfrit; POOK, Karl-Heinz. Pilosin und epiisopilosin. Justus Liebigs Annalen der Chemie, [S.l.], v. 1.973, n. 9, p. 1.476-1.479, 1973.

MANS, Dennis; ROCHA, Adriana B.; SCHWARTSMANN, Gilberto. Anti-cancer drug discovery and development in Brazil: targeted plant collection as a rational strategy to acquire candidate anti-cancer compounds. The Oncologist, [S.l.], v. 5, n. 3, p. 185-198, 2000.

MALAYSIA, Preena Sidhu. Endangered Jaborandi. British Dental Journal, [S.l.], v. 217, n. 1, jul. 2014.

MARIN, Fernis J. et al. Estudo Fitoquímico e Avaliação da Atividade Antioxidante e Leishmanicida da Espécie Pilocarpus alvaradoi (Rutaceae). Información Tecnológica, [S.l.], v. 29, n. 3, p. 177-186, 2018.

MARTINELLI, Gustavo; MORAES, Miguel Avila. Livro vermelho da flora do Brasil. 1. ed. Rio de Janeiro: Andrea Jakobsson; Instituto de Pesquisas Jardim Botânico do Rio de Janeiro, 2013. 1102 p. Disponível em: http://cncflora.jbrj.gov.br/arquivos/arquivos/pdfs/LivroVerme lho.pdf. Acesso em: 2 fev. 2020. 
MOURA, E. F. et al. Genetic diversity in a jaborandi (Pilocarpus microphyllus Stapf.) germplasm bank assessed by RAPD markers. Embrapa Amazônia Oriental, [S.l.], artigo em periódico indexado (ALICE), 2005.

NOGUEIRA, Fabíola Leite; SAKATA, Rioko Kimiko. Sedação paliativa do paciente terminal. Revista Brasileira de Anestesiologia, [S.I.], v. 62, n. 4, p. 580-92, 2012.

OLIVEIRA, Ana Claudia Dias et al. Os dez anos da Política Nacional de Plantas Medicinais e Fitoterápicos (PNPMF) e os principais entraves da cadeia produtiva de extratos vegetais $e$ medicamentos fitoterápicos no Brasil. Revista Fitos, Rio de Janeiro, v., 10, n. 2, p. 95-219, 2016.

OMS - ORGANIZAÇÃO MUNDIAL DA SAÚDE. Global Innovation Index 2018. Disponível em: https://www.wipo.int/pressroom/en/ar ticles/2018/article_0005.html. Acesso em: 7 jan. 2020.

PEREIRA, Renata Junqueira; CARDOSO, Maria das Graças. Metabólitos secundários vegetais e benefícios antioxidantes. Journal of Biotechnology and Biodiversity, [S.l.], v. 3, n. 4, 2012.

PINHEIRO, Claudio Urbano B. Extractivism, cultivation and privatization of jaborandi (Pilocarpus microphyllus Stapf ex Holm; Rutaceae) in Maranhão, Brazil. Acta Botânica Brasilica, [S.l.], v. 16, n. 2, p. 141-150, 2002.

QUINTELLA, Cristina Maria et al. Prospecção tecnológica como uma ferramenta aplicada em ciência e tecnologia para se chegar à inovação. Revista Virtual de Química, [S.1.], v. 3, n. 5, p. 406-415, 2011.

ROCHA, Jefferson A. et al. Anthelmintic, antibacterial and cytotoxicity activity of imidazole alkaloids from Pilocarpus microphyllus leaves. Phytotherapy Research, [S.l.], v. 31, n. 4, p. 624-630, 2017.

RODRIGUES, Waldecy; BARBOSA, Gislane Ferreira. Plantas Medicinais: Uma Alternativa Econômica para Conservação do Cerrado Brasileiro? Informe Gepec, [S.I.], v. 16, n. 1, p. 160-175, 2012.

SANTOS, Ana Paula et al. Analysis of the volatile oil from Pilocarpus pennatifolius Lemmaire

(Rutaceae) leaves by GC-MS. Flavour and Fragrance Journal, [S.1.], v. 19, n. 4, p. 325-326, 2004.

SANTOS, Jane Lucia Silva; HANSEN, Peter Bent. Estratégias em parques tecnológicos: Uma análise da produção científica na base Web of Science. Internacional Journal of Knowledge Engineering and Management (IJKEM), [S.l.], v. 5, n. 12, p. 29-48, 2016.

SALLES, Luisa de Andrade et al. Pilocarpine accumulation on Pilocarpus pennatifolius tissue culture. Revista Brasileira de Ciências Farmacêuticas, [S.I.], v. 40, n. 3, p. 437-439, 2004.

SAWAYA, Alexandra Christine Helena Frankland et al. Screening species of Pilocarpus (Rutaceae) as sources of pilocarpine and other imidazole alkaloids. Genetic Resources and Crop Evolution, [S.l.], v. 58, n. 3, p. 471-480, 2011.

SIDHU, Preena. Endangered jaborandi. British Dental Journal, [S.I.], v. 217, n. 1, p. 2, 2014.

SKORUPA, Ladislau. Espécies de Pilocarpus Vahl (Rutaceae) da Amazônia brasileira. Acta Amazonica, [S.1.], v. 30, n. 1, p. 59-70, 2000.

SILVA, J. C. R.; RAO, V. S. N. Involvement of serotonin and eicosanoids in the rat paw oedema response to the essential oil of Pilocarpus spicatus. Mediators of Inflammation, [S.l.], v. 1, n. 3, p. 167-169, 1992. 
SILVA, Cinara Vasconcelos da et al. Estudo fitoquímico e avaliação in vitro da atividade antiTrypanosoma cruzi cepa Y de Pilocarpus spicatus St. Hil.(Rutaceae). Revista Brasileira de Plantas Medicinais, v. 16, n. 4, p. 812-818, 2014.

SIMÕES, C. M. O. et al. Farmacognosia: da planta ao medicamento. 6. ed. Porto Alegre: Editora da UFRGS, 2007. 1.104 p.

SOUZA FILHO, João Pessoa de et al. A evolução do mercado farmacêutico brasileiro no tratamento do glaucoma nos últimos 30 anos. Arquivos Brasileiros de Oftalmologia, [S.l.], 2003.

TEDESCHI, E. et al. The Isolation of Pure Pilosine and Epi-Isopilosine from Leaves of Pilocarpus Jaborandi. Israel Journal of Chemistry, [S.I.], v. 11, n. 5, p. 731-733, 1973.

TUNMANN, O.; JENZER, R. Zur Anatomie der Blüten von Pilocarpus pennatifolius Lem. und Erythroxylon coca Lam. Archiv der Pharmazie, [S.I.], v. 248, n. 2-8, p. 514-519, 1910.

VERAS, Leiz Maria. et al. Activity of epiisopiloturine against Schistosoma mansoni. Current Medicinal Chemistry, [S.l.], v. 19, n. 13, p. 2.051-2.058, 2012.

VYSOTSKAIA, O. S.; MUSHINSKAIA, Skh; SHOSTENKO, IuV. Quantitative determination of alkaloids of Pilocarpus in plant raw materials and in semiproducts of pilocarpine production. Farmatsevtychnyi Zhurnal, [S.l.], v. 21, n. 4, p. 61-65, 1966.

WATERMAN, Peter G. Alkaloids of the Rutaceae: their distribution and systematic significance. Biochemical Systematics and Ecology, [S.I.], v. 3, n. 3, p. 149-180, 1975.

WIPO - WORLD INTELLECTUAL PROPERTY ORGANIZATION. Classificação Internacional de Patentes (IPC). [2018]. Disponível em: http://www.wipo.int/classifications/ipc/en/. Acesso em: 12 fev. 2020.

WIPO - WORLD INTELLECTUAL PROPERTY ORGANIZATION. [2020]. Disponível em: https:// patentscope.wipo.int/search/pt/structuredSearch.jsf. Acesso em: 10 fev. 2020.

\section{Sobre os Autores}

\section{Cleiton Barroso Bittencourt}

E-mail: cleiton_court@hotmail.com

Doutorando em Biotecnologia Vegetal pela Universidade Federal de Lavras (UFLA).

Endereço profissional: Universidade Federal de Lavras (UFLA), Campus Universitário, s/n, Lavras, MG. CEP: 37200-000.

\section{Maria Gracelia Paiva Nascimento}

E-mail: graceliapaiva@gmail.com

Doutoranda em Desenvolvimento e Meio Ambiente (PRODEMA/UFPI/TROPEN) pela Universidade Federal do Piauí (UFPI).

Endereço profissional: Av. Universitária, n. 1.310, Bairro Ininga, Teresina, PI. CEP: 64049-550. 


\section{Jéssica Cristina Moraes de Araújo}

E-mail: jeeh.cristina@hotmail.com

Mestranda em Biotecnologia pela Universidade Federal do Delta do Parnaíba (UFDPar).

Endereço profissional: Universidade Federal do Delta do Parnaíba (UFDPar), Av. São Sebastião, n. 2.819, Nossa

Sra. de Fátima, Parnaíba, PI. CEP: 64202-020.

\section{Davi Nascimento Costa}

E-mail: daveoficial123@gmail.com

Graduando em Ciências Biológicas pela Universidade Federal do Delta do Parnaíba (UFDPar).

Endereço profissional: Universidade Federal do Delta do Parnaíba (UFDPar), Av. São Sebastião, n. 2.819, Nossa

Sra. de Fátima, Parnaíba, PI. CEP: 64202-020.

\section{Juelina Oliveira dos Santos}

E-mail: jjuelina@hotmail.com

Mestre em Biotecnologia pela Universidade Federal do Piauí (UFPI) em 2017.

Endereço profissional: Universidade Federal do Delta do Parnaíba (UFDPar), Av. São Sebastião, n. 2.819, Nossa

Sra. de Fátima, Parnaíba, PI. CEP: 64202-020.

\section{Ivanilza Moreira de Andrade}

E-mail: ivanilzaandrade@hotmail.com

Doutora em Botânica pela Universidade Estadual de Feira de Santana (UEFS) em 2006. Atualmente é docente da Universidade Federal do Delta do Parnaíba (UFDPar).

Endereço profissional: Universidade Federal do Delta do Parnaíba (UFDPar), Av. São Sebastião, n. 2.819, Nossa Sra. de Fátima, Parnaíba, PI. CEP: 64202-020. 